\title{
Singular value decomposition applied to compact binary coalescence gravitational-wave signals
}

\author{
Kipp Cannon, ${ }^{1, *}$ Adrian Chapman, ${ }^{1}$ Chad Hanna, ${ }^{1, \dagger}$ Drew Keppel, ${ }^{1,2, \sharp}$ Antony C. Searle, ${ }^{1, \S}$ and Alan J. Weinstein ${ }^{1, \|}$ \\ ${ }^{1}$ LIGO Laboratory, California Institute of Technology, Pasadena, California 91125, USA \\ ${ }^{2}$ Theoretical Astrophysics, California Institute of Technology, Pasadena, California 91125, USA
}

(Received 18 May 2010; published 12 August 2010)

\begin{abstract}
We investigate the application of the singular value decomposition to compact-binary, gravitationalwave data-analysis. We find that the truncated singular value decomposition reduces the number of filters required to analyze a given region of parameter space of compact-binary coalescence waveforms by an order of magnitude with high reconstruction accuracy. We also compute an analytic expression for the expected signal loss due to the singular value decomposition truncation.
\end{abstract}

DOI: 10.1103/PhysRevD.82.044025

PACS numbers: 04.30.- w, 07.05.Kf

\section{INTRODUCTION}

The coalescence of compact binaries composed of neutron stars and or black holes is a promising source of gravitational radiation for ground-based gravitationalwave $(\mathrm{GW})$ detectors. The mass parameters of the GW signal are not known a priori. In order to detect GW from compact-binary coalescence (CBC) events, a large number of filter templates are required to probe the continuous component mass parameter space, $\left(m_{1}, m_{2}\right)$, of possible CBC signals in the detector data to high fidelity $[1,2]$. Template waveforms are distributed in the space such that there is a small maximum loss of signal-to-noise ratio (SNR) (called the "minimal match") due to the mismatch between an arbitrary point in the mass parameter space and the nearest discrete point of the template bank. A standard choice for the minimal match is $97 \%$, which, for a hexagonally tiled, flat, two-dimensional manifold, corresponds to neighboring templates that have greater than $95 \%$ overlap.

This redundancy implies that correlated calculations are required to filter the data with these templates. The singular value decomposition (SVD) can be used to eliminate these correlations by producing orthogonal basis vectors that can be used for filtering and reconstructing the original template bank.

This work will describe how to reduce the computational redundancy in filtering the $\mathrm{CBC}$ signal parameter-space in order to more efficiently infer whether or not a GW is present. Specifically, we will explore a purely numerical technique using the SVD to reduce the number of templates required to search the data. We note that others have applied the use of SVD to GW data-analysis to analyze optimal GW burst detection [3,4] and coherent networks of detectors [5]. We also note that significant work has been done to analytically reduce the computational filtering

\footnotetext{
*kipp.cannon@ligo.org

†chad.hanna@ligo.org

*drew.keppel@ligo.org

\$antony.searle@ligo.org

"
}

burden using interpolation for certain template waveforms $[6,7]$.

This paper is organized as follows. First, we describe the framework for CBC filtering in the context of vector inner products. Next we introduce the SVD as a way to reduce the number of filters required to approximately compute those inner products. We then derive an expression for the expected SNR loss in terms of the singular values. Finally, we demonstrate the application of this method to a set of CBC waveforms corresponding to binary neutron star (BNS) coalescences.

\section{METHOD}

\section{A. Matched filtering}

CBC searches employ matched filtering as the first step in locating a GW signal [8]. The optimal filtering strategy weights both the detector output and template waveform by the inverse of the amplitude spectral density of the detector noise, a process called "whitening". Representing both the whitened data and the $\alpha$ th whitened template waveform as discretely sampled time series, $\vec{s}=\left\{s_{i}\right\}$ and $\vec{h}_{\alpha}=\left\{h_{\alpha i}\right\}$, respectively, the output of the matched filter at a specific point in time is given by the vector inner product

$$
\rho_{\alpha}=\vec{h}_{\alpha}^{*} \cdot \vec{s}
$$

In searches for $\mathrm{GWs}$ from $\mathrm{CBC}$ sources, the signals being sought are chirping sinusoids with an unknown phase. The search over phase is accomplished through the use of complex-valued templates where $\Re \vec{h}_{\alpha}$ contains the cosinelike phase and $\mathfrak{s}_{\alpha}$ contains the sinelike phase. The filter output can be maximized over template phase by evaluating $\left|\rho_{\alpha}\right|$.

In the absence of a GW signal, the whitened detector data consists only of noise, $\vec{n}=\left\{n_{i}\right\}$, and is a stationary, zero-mean, unit-variance, Gaussian random process, so

$$
\begin{aligned}
\left\langle n_{i}\right\rangle & =0, \\
\left\langle n_{i} n_{j}\right\rangle & =\delta_{i j},
\end{aligned}
$$

where \langle\rangle denotes the ensemble average. When the template 
waveforms are normalized such that $\Re \vec{h}_{\alpha} \cdot \Re \vec{h}_{\alpha}=\Im \vec{h}_{\alpha}$. $\Im \vec{h}_{\alpha}=1$, (2) yields

$$
\begin{aligned}
\left\langle\vec{h}_{\alpha}^{*} \cdot \vec{n}\right\rangle & =0, \\
\left\langle\left(\Re \vec{h}_{\alpha} \cdot \vec{n}\right)^{2}\right\rangle & =\left\langle\left(\Im \vec{\jmath}_{\alpha} \cdot \vec{n}\right)^{2}\right\rangle=1 .
\end{aligned}
$$

When (3) is true, $\rho_{\alpha}$ is called the SNR and indicates how likely it is that a signal is present in the data at that point in time [9].

As explained in Sec. I, $\vec{h}_{\alpha} \cdot \vec{h}_{\alpha^{\prime}}>0.95$ for adjacent templates. For those templates, $\rho_{\alpha}$ and $\rho_{\alpha^{\prime}}$ differ by, at most, $5 \%$. This suggests the existence of an approximation scheme that would allow the SNRs to be computed to reasonable accuracy without explicitly evaluating all the template inner products. Next, we will look at how the truncated SVD can be used to replace the template bank with an approximate, lower-rank, orthogonal basis from which the SNRs can be reconstructed.

\section{B. Reducing the number of filters with truncated singular value decomposition}

The waveforms are parameterized by their component masses and we denote the $\alpha$ th template waveform of the $M$ templates required to search a given parameter space as $\vec{h}_{\alpha}=\left\{h\left(m_{1}, m_{2}, t_{i}\right)\right\}$. Rather than filter the data with $N=$ $2 M$ real-valued filters ( $M$ complex-valued filters), we linearly combine the output of a basis set of fewer, realvalued, filters, $\vec{u}_{\mu}$, to reproduce $\rho_{\alpha}$ to the desired accuracy, $\rho_{\alpha}^{\prime}$. The goal is to have

$$
\rho_{\alpha}^{\prime}=\sum_{\mu=1}^{N^{\prime}} A_{\alpha \mu}\left(\vec{u}_{\mu} \cdot \vec{s}\right)
$$

where $\mathbf{A}$ is the complex-valued reconstruction matrix we wish to find and the number of inner products is reduced from $N$ to $N^{\prime}$. In order to find the basis vectors, $\vec{u}_{\mu}$, we use the SVD of the real-valued template matrix, $\mathbf{H}$

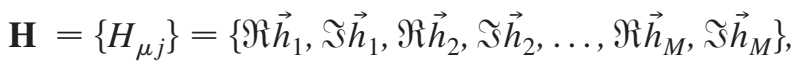

where $\mu$ identifies rows of $\mathbf{H}$ and indexes the filter number, and $j$ identifies the columns of $\mathbf{H}$ and indexes sample points. In this definition, the row vectors $\vec{H}_{(2 \alpha-1)}$ and $\vec{H}_{(2 \alpha)}$ are, respectively, the real and imaginary parts of the $\alpha$ th complex waveform, $\vec{h}_{\alpha}$. An illustrative template matrix can be seen in Fig. 1.

The SVD factors a matrix such that ([10] Sec. 14.4)

$$
H_{\mu j}=\sum_{\nu=1}^{N} v_{\mu \nu} \sigma_{\nu} u_{\nu j},
$$

where $\mathbf{v}$ is an orthonormal matrix of reconstruction coefficients whose columns, $v_{\mu \nu}$, satisfy

$$
\sum_{\mu} v_{\mu \nu} v_{\mu \lambda}=\delta_{\nu \lambda}
$$
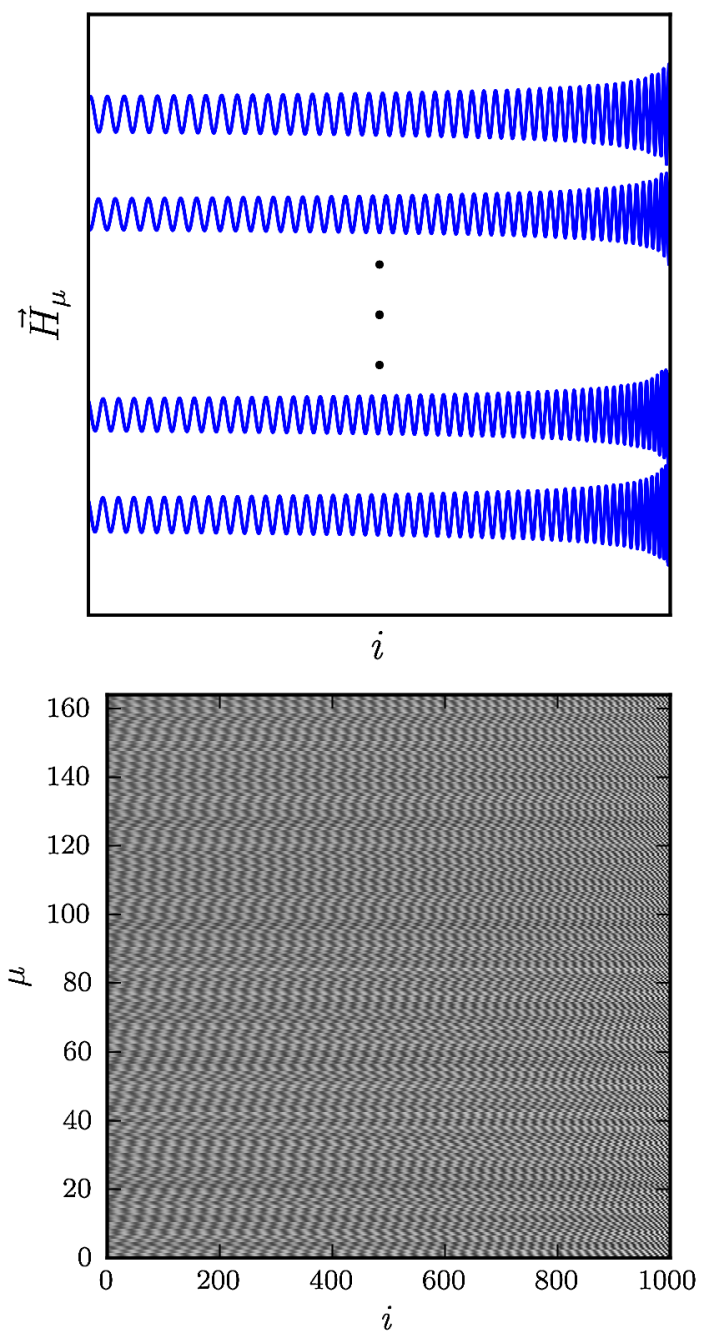

FIG. 1 (color online). An example template matrix, H. Top: An illustration of how the input template time series is packed into the template matrix. Bottom: The matrix of the template time series where the $y$-axis indicates the template waveform and the $x$-axis indicates the time samples. It should be noted that these waveforms have been shortened and have not been whitened for illustrative purposes.

$\vec{\sigma}$ is a vector of singular values ranked in order of importance in reconstructing the $\mathbf{H}$, and $\mathbf{u}$ is a matrix of orthonormal bases (e.g., an illustration can be found in Fig. 2) whose rows are basis vectors, $\vec{u}_{\mu}$, satisfying

$$
\sum_{j} u_{\mu j} u_{\nu j}=\delta_{\mu \nu}
$$

However, since a search for CBC signals only needs waveform accuracies of a few percent to be successful, it is possible to make an approximate reconstruction of $\mathbf{H}$

$$
H_{\mu j} \approx H_{\mu j}^{\prime}:=\sum_{\nu=1}^{N^{\prime}} v_{\mu \nu} \sigma_{\nu} u_{\nu j},
$$

where $N^{\prime}<N$. This reduces the number of rows of $\mathbf{u}$ used in the reconstruction. We create a new basis matrix $\mathbf{u}=$ $\left\{u_{\nu j}\right\}=\left\{\vec{u}_{1}, \vec{u}_{2}, \ldots \vec{u}_{N^{\prime}}\right\}$, where $\nu$ indexes the filter number, 

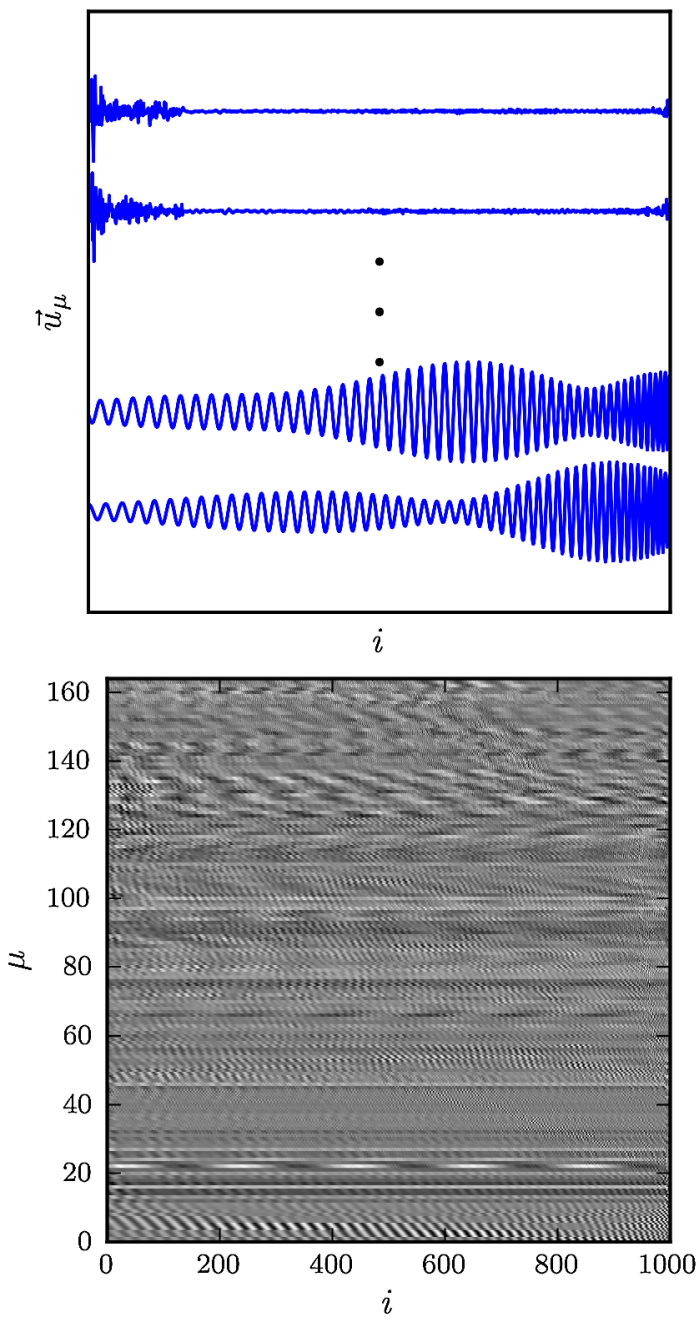

FIG. 2 (color online). An example basis matrix, u. Top: An illustration of the resulting orthonormal basis vectors ordered from most to least important (bottom to top) in reconstructing $\mathbf{H}$. Bottom: The matrix of basis waveforms produced by the SVD. The $y$-axis indexes the basis waveforms and the $x$-axis indicates time samples. It should be noted that these basis vectors have been computed from shortened, nonwhitened template waveforms as mentioned in Fig. 1 purely for illustrative purposes.

$j$ indexes sample points, and we have discarded the basis vectors that look least like the template waveforms (i.e. with the lowest singular values). We can write (4) as

$$
\begin{aligned}
\rho_{\alpha}^{\prime} & =\left(\vec{H}_{(2 \alpha-1)}^{\prime}-\mathrm{i} \vec{H}_{(2 \alpha)}^{\prime}\right) \cdot \vec{s} \\
& =\sum_{\nu=1}^{N^{\prime}}\left(v_{(2 \alpha-1) \nu} \sigma_{\nu}-\mathrm{i} v_{(2 \alpha) \nu} \sigma_{\nu}\right)\left(\vec{u}_{\nu} \cdot \vec{s}\right),
\end{aligned}
$$

where we have made use of the packing of $\mathbf{H}$ (5) and (9).

\section{Reconstruction accuracy}

As we are not reconstructing the original template waveforms exactly, there will be some inherent mismatch be- tween $\vec{H}_{\mu}^{\prime}$ and $\vec{H}_{\mu}$. We want to know the expected fractional SNR we will lose because of this difference.

As stated previously, the inner product of a (normalized) template waveform, $\vec{H}_{\mu}$, with itself is

$$
\vec{H}_{\mu} \cdot \vec{H}_{\mu}=1=\sum_{\nu=1}^{N} v_{\mu \nu}^{2} \sigma_{\nu}^{2}
$$

where, in the second line, we have made use of the orthogonality of basis vectors (8). A similar relation can be found for the inner product of the reconstructed waveform, $\vec{H}_{\mu}^{\prime}$, with itself

$$
\vec{H}_{\mu}^{\prime} \cdot \vec{H}_{\mu}^{\prime}=\sum_{\nu=1}^{N^{\prime}} v_{\mu \nu}^{2} \sigma_{\nu}^{2}=1-\sum_{\nu=N^{\prime}+1}^{N} v_{\mu \nu}^{2} \sigma_{\nu}^{2} .
$$

Because of the orthogonality of the basis vectors (8), the inner product between a template waveform, $\vec{H}_{\mu}$, with a reconstructed waveform, $\vec{H}_{\nu}^{\prime}$, is

$$
\vec{H}_{\mu} \cdot \vec{H}_{\nu}^{\prime}=\vec{H}_{\mu}^{\prime} \cdot \vec{H}_{\nu}=\vec{H}_{\mu}^{\prime} \cdot \vec{H}_{\nu}^{\prime} .
$$

In addition, the two phases of the templates, which are packed adjacently in $\mathbf{H}$ (5), are orthogonal

$$
\vec{H}_{(2 \mu-1)} \cdot \vec{H}_{(2 \mu)}=\sum_{\nu=1}^{N} v_{(2 \mu-1) \nu} v_{(2 \mu) \nu} \sigma_{\nu}^{2}=0 .
$$

This implies that the inner product of the two phases of the approximate waveforms are given as

$$
\begin{aligned}
\vec{H}_{(2 \mu-1)}^{\prime} \cdot \vec{H}_{(2 \mu)}^{\prime} & =\sum_{\nu=1}^{N^{\prime}} v_{(2 \mu-1) \nu} v_{(2 \mu) \nu} \sigma_{\nu}^{2} \\
& =-\sum_{\nu=N^{\prime}+1}^{N} v_{(2 \mu-1) \nu} v_{(2 \mu) \nu} \sigma_{\nu}^{2} .
\end{aligned}
$$

The average fractional SNR loss, $\delta \rho_{\alpha} / \rho_{\alpha}$, between a template waveform and the two phases of the same reconstructed waveform is given by

$$
\frac{\delta \rho_{\alpha}}{\rho_{\alpha}}:=1-\frac{\left|\rho_{\alpha}^{\prime}\right|}{\left|\rho_{\alpha}\right|} .
$$

The following derives the mismatch in terms the of components we truncate from the SVD. First we compute these terms for a given signal waveform, $\vec{s}=\Re\left(A \mathrm{e}^{\mathrm{i} \phi} \vec{h}_{\alpha}\right)$, with phase, $\phi$. The SNR from the exact waveform, $\left|\rho_{\alpha}(\phi)\right|$, is given as

$$
\begin{aligned}
& \left|\rho_{\alpha}(\phi)\right|=\left[\left(\frac{\Re \vec{h}_{\alpha} \cdot A \Re\left(\mathrm{e}^{\mathrm{i} \phi} \vec{h}_{\alpha}\right)}{\sqrt{\Re \vec{h}_{\alpha} \cdot \Re \vec{h}_{\alpha}}}\right)^{2}\right. \\
& \left.+\left(\frac{\mathfrak{s} \vec{h}_{\alpha} \cdot A \Re\left(\mathrm{e}^{\mathrm{i} \phi} \vec{h}_{\alpha}\right)}{\sqrt{\mathfrak{\Im} \vec{h}_{\alpha} \cdot \mathfrak{s} \vec{h}_{\alpha}}}\right)^{2}\right]^{1 / 2}=A,
\end{aligned}
$$

in which we have used (11) and (14). The SNR from the 
approximate waveform, $\left|\rho_{\alpha}^{\prime}\right|$, is given as

$$
\begin{aligned}
\left|\rho_{\alpha}^{\prime}(\phi)\right|= & {\left[\left(\frac{\Re \vec{h}_{\alpha}^{\prime} \cdot A \Re\left(\mathrm{e}^{\mathrm{i} \phi} \vec{h}_{\alpha}\right)}{\sqrt{\Re \vec{h}_{\alpha}^{\prime} \cdot \Re \vec{h}_{\alpha}^{\prime}}}\right)^{2}\right.} \\
& \left.+\left(\frac{\Im \vec{h}_{\alpha}^{\prime} \cdot A \Re\left(\mathrm{e}^{\mathrm{i} \phi} \vec{h}_{\alpha}\right)}{\sqrt{\mathfrak{\Im} \vec{h}_{\alpha}^{\prime} \cdot \Im \vec{h}_{\alpha}^{\prime}}}\right)^{2}\right]^{1 / 2}
\end{aligned}
$$

We can expand (18) using the packing of $\mathbf{H}$ (5), (12), (13), and (15) to

$$
\begin{aligned}
\left|\rho_{\alpha}^{\prime}(\phi)\right|= & A\left[\cos ^{2} \phi\left(1-\sum_{\mu=N^{\prime}+1}^{N} v_{(2 \alpha-1) \mu}^{2} \sigma_{\mu}^{2}\right)\right. \\
& +\sin ^{2} \phi\left(1-\sum_{\mu=N^{\prime}+1}^{N} v_{(2 \alpha) \mu}^{2} \sigma_{\mu}^{2}\right) \\
& +4 \cos \phi \sin \phi \sum_{\mu=N^{\prime}+1}^{N} v_{(2 \alpha-1) \mu} v_{(2 \alpha) \mu} \sigma_{\mu}^{2} \\
& +\sin ^{2} \phi \frac{\left(\sum_{\mu=N^{\prime}+1}^{N} v_{(2 \alpha-1) \mu} v_{(2 \alpha) \mu} \sigma_{\mu}^{2}\right)^{2}}{1-\sum_{\mu=N^{\prime}+1}^{N} v_{(2 \alpha-1) \mu}^{2} \sigma_{\mu}^{2}} \\
& \left.+\cos ^{2} \phi \frac{\left(\sum_{\mu=N^{\prime}+1}^{N} v_{(2 \alpha-1) \mu} v_{(2 \alpha) \mu} \sigma_{\mu}^{2}\right)^{2}}{1-\sum_{\mu=N^{\prime}+1}^{N} v_{(2 \alpha) \mu}^{2} \sigma_{\mu}^{2}}\right]^{1 / 2}
\end{aligned}
$$

Let us look at the higher order sums in (19). The sums $\sum_{\nu=N^{\prime}+1}^{N} v_{\mu \nu}^{2} \sigma_{\nu}^{2}$, which are also found in (12), represent the power of vector $\vec{H}_{\mu}$ lost through the truncation of the SVD. These sums must be less than $1, \sum_{\nu=N^{\prime}+1}^{N} v_{\mu \nu}^{2} \sigma_{\nu}^{2}<$ 1. However, since the objective is for the approximation to be such that $\left\|\vec{H}_{\mu}-\vec{H}_{\mu}^{\prime}\right\| \sim 1 \%$, we expect

$$
\sum_{\nu=N^{\prime}+1}^{N} v_{\mu \nu}^{2} \sigma_{\nu}^{2} \ll 1
$$

and we can therefore drop terms that are higher than first order in these sums. Additionally,

$$
\begin{aligned}
\left|\sum_{\nu=N^{\prime}+1}^{N} v_{\mu \nu} v_{\mu^{\prime} \nu} \sigma_{\nu}^{2}\right| & \leq \sqrt{\left(\sum_{\nu=N^{\prime}+1}^{N} v_{\mu \nu}^{2} \sigma_{\nu}^{2}\right)\left(\sum_{\nu=N^{\prime}+1}^{N} v_{\mu^{\prime} \nu}^{2} \sigma_{\nu}^{2}\right)} \\
& \ll 1
\end{aligned}
$$

This means (19) is approximately

$$
\begin{aligned}
\left|\rho_{\alpha}^{\prime}(\phi)\right| \approx & A\left[1-\frac{1}{2} \cos ^{2} \phi \sum_{\mu=N^{\prime}+1}^{N} v_{(2 \alpha-1) \mu}^{2} \sigma_{\mu}^{2}\right. \\
& -\frac{1}{2} \sin ^{2} \phi \sum_{\mu=N^{\prime}+1}^{N} v_{(2 \alpha) \mu}^{2} \sigma_{\mu}^{2} \\
& \left.+2 \cos \phi \sin \phi \sum_{\mu=N^{\prime}+1}^{N} v_{(2 \alpha-1) \mu} v_{(2 \alpha) \mu} \sigma_{\mu}^{2}\right] .
\end{aligned}
$$

As physical signals will arrive in the detectors with random phases, we now average over the phase, $\phi$, using

$$
\left|\rho_{\alpha}\right|:=\frac{1}{2 \pi} \int_{0}^{2 \pi}\left|\rho_{\alpha}(\phi)\right| d \phi,
$$

resulting in

$\left|\rho_{\alpha}\right|=A$,

$\left|\rho_{\alpha}^{\prime}\right|=A\left[1-\frac{1}{4} \sum_{\mu=N^{\prime}+1}^{N}\left(v_{(2 \alpha-1) \mu}^{2}+v_{(2 \alpha) \mu}^{2}\right) \sigma_{\mu}^{2}\right]$

Substituting (24a) and (24b) in (16), we find the average fractional SNR loss for the $\alpha$ th template

$$
\frac{\delta \rho_{\alpha}}{\rho_{\alpha}}=\frac{1}{4} \sum_{\mu=N^{\prime}+1}^{N}\left(v_{(2 \alpha-1) \mu}^{2}+v_{(2 \alpha) \mu}^{2}\right) \sigma_{\mu}^{2} .
$$

The expected fractional SNR loss can be computed by averaging over the waveforms in the template bank using

$$
\left\langle\frac{\delta \rho}{\rho}\right\rangle:=\frac{1}{M} \sum_{\alpha=1}^{M} \frac{\delta \rho_{\alpha}}{\rho_{\alpha}} .
$$

Combining (25) with (26), remembering $M=N / 2$, and using the orthogonality of reconstruction coefficients (7), we get

$$
\left\langle\frac{\delta \rho}{\rho}\right\rangle=\frac{1}{2 N} \sum_{\mu=N^{\prime}+1}^{N} \sigma_{\mu}^{2}
$$

It is not surprising that the expected fractional SNR loss is proportional to the square of the Frobenius norm of the truncation error of $\mathbf{H}$

$$
\left\|\mathbf{H}-\mathbf{H}^{\prime}\right\|_{2}^{2}=\sum_{\mu, j}\left(H_{\mu j}-H_{\mu j}^{\prime}\right)^{2}=\sum_{\nu=N^{\prime}+1}^{N} \sigma_{\nu}^{2} .
$$

The expected fractional SNR loss, $\langle\delta \rho / \rho\rangle$, can be used as a threshold for deciding how many basis vectors to keep in the truncated SVD reconstruction of the template matrix. For detection purposes, we want $\langle\delta \rho / \rho\rangle$ to be less than the minimal match of the template bank. 


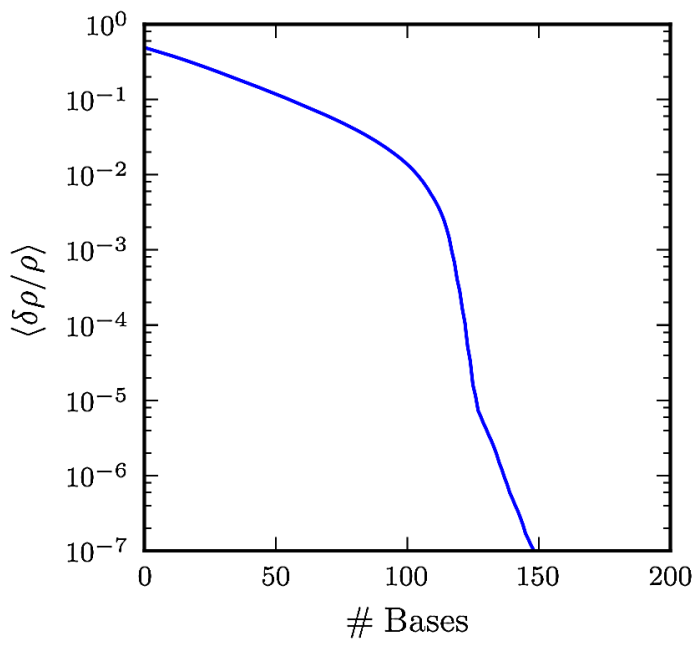

FIG. 3 (color online). The expected fractional SNR loss, $\langle\delta \rho / \rho\rangle$, given by (27) as a function of the number of basis vectors we retain (out of $N=912$ ). The region $\langle\delta \rho / \rho\rangle>10 \%$ should be ignored as the Taylor expansion of the fractional SNR loss in (22) is not valid in that regime.

\section{APPLICATION TO COMPACT BINARY COALESCENCE GRAVITATIONAL-WAVE SIGNALS}

We apply the above procedure to BNS waveforms with chirp masses $1.125 M_{\odot} \leq M_{c}<1.240 M_{\odot}$ and component masses $1 M_{\odot} \leq m_{1}, m_{2}<3 M_{\odot}$. The number of templates required to hexagonally cover this range in parameters using a minimal match of $96.8 \%$ is $M=456$, which implies a total number of filters $N=912$. These nonspinning waveforms were produced to $3.5 \mathrm{PN}$ order[11], sampled at $2048 \mathrm{~Hz}$, up to the Nyquist frequency of $1024 \mathrm{~Hz}$. The last

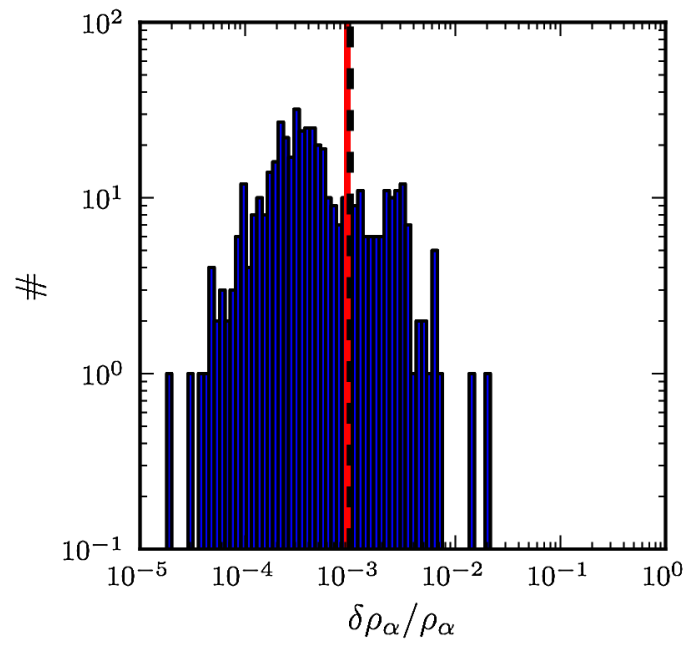

FIG. 4 (color online). Histogram of measured fractional SNR loss, $\delta \rho_{\alpha} / \rho_{\alpha}$, where we have chosen a random value of $\phi$ for each template. The mean value predicted by (27), shown as the dashed black line, matches the measured mean shown as the solid line.
10 seconds of each waveform, whitened with the initial LIGO amplitude spectral density, were used to construct H.

In Fig. 3, we plot $\langle\delta \rho / \rho\rangle$ as a function of the number of basis vectors kept. If we require that $\langle\delta \rho / \rho\rangle=10^{-3}$, we find we can reduce the number of filters in the above template bank from $N=912$ to $N^{\prime}=118$, about an order of magnitude reduction in the number of filters.

In Fig. 4 , we show how $\langle\delta \rho / \rho\rangle$ compares to the actual distribution of $\delta \rho_{\alpha} / \rho_{\alpha}$, where we have chosen random values of $\phi$ for each template. We find it is a good measure of the expected fractional loss of SNR.

We have investigated how generic this reduction of filters is for other regions of CBC mass parameter space (e.g., regions of parameter space with larger component masses), and find the reduction to be similar. We tested this by generating a template bank with a $96.8 \%$ minimal match, component masses between $1 M_{\odot}$ and $34 M_{\odot}$, and total mass below $35 M_{\odot}$. We then ordered the templates by chirp mass, split the template bank up into patches of $M=$ 456 templates, and computed the SVD for these patches.

We can include larger portions of parameter space in the SVD by including more templates such that the number of templates is smaller than the number of time-samples per template. However the computational cost of the SVD of an $N \times L$ matrix with $N \leq L$ grows as $\mathcal{O}\left(L N^{2}\right)$, thus, including more templates nonlinearly increases the cost. Another complication is that waveforms further apart in parameter space have smaller overlap. This will result in more basis vectors being required to reconstruct the waveforms to the same accuracy. Therefore, including larger portions of parameter space in a single SVD computation will result in diminishing returns for the computational cost. We propose to address this issue, as above, by breaking up the parameter space into patches for which we can independently compute the SVD, although how best to do this is beyond the scope of the present work.

\section{CONCLUSION}

We have investigated how the SVD can be used to reduce the number of filters needed when analyzing GW data for CBC signals. We have found the number of filters required to matched filter these template banks can be reduced by about an order of magnitude through truncating the SVD of these waveforms. This result differs from other work that models CBC GW signals in approximate ways [12-14] by starting with an exact representation of the desired template family and producing a rigorous approximation with a tunable accuracy.

We plan to explore several topics in future works. Among these are the derivation of a composite detection statistic using only the SVD coefficients in order to minimize the computational costs associated with reconstruction and the interpolation of signals not in the original template set. 


\section{ACKNOWLEDGMENTS}

The authors would like to acknowledge the support of the LIGO Lab, NSF grants PHY-0653653 and PHY0601459, and the David and Barbara Groce Fund at the California Institute of Technology. LIGO was constructed by the California Institute of Technology and
Massachusetts Institute of Technology with funding from the National Science Foundation and operates under cooperative agreement PHY-0757058. The authors also thank Stephen Privitera and Ik Siong Heng for useful comments and discussions on this manuscript. This paper has LIGO Document Number LIGO-P1000037-v2.
[1] B. J. Owen, Phys. Rev. D 53, 6749 (1996).

[2] B. J. Owen and B.S. Sathyaprakash, Phys. Rev. D 60, 022002 (1999).

[3] P. R. Brady and S. Ray-Majumder, Classical Quantum Gravity 21, S1839 (2004).

[4] I.S. Heng, Classical Quantum Gravity 26, 105005 (2009).

[5] L. Wen, Int. J. Mod. Phys. D 17, 1095 (2008).

[6] R. P. Croce, T. Demma, V. Pierro, I. M. Pinto, and F. Postiglione, Phys. Rev. D 62, 124020 (2000).

[7] A. S. Mitra, S. V. Dhurandhar, and L. S. Finn, Phys. Rev. D 72, 102001 (2005).

[8] B. A. Allen, W. G. Anderson, P. R. Brady, D. A. Brown, and J. D. E. Creighton, arXiv:gr-qc/0509116.

[9] L. A. Wainstein and V. D. Zubakov, Extraction of Signals
From Noise (Prentice-Hall, Englewood Cliffs, NJ, 1962).

[10] M. Galassi, J. Davies, J. Theiler, B. Gough, G. Jungman, P. Alken, M. Booth, and F. Rossi, GNU Scientific Library Reference Manual (Network Theory Ltd., United Kingdom, 2009), 3rd ed, for version 1.12.

[11] LSC Algorithms LibraryLIGO Scientific Collaboration, https://www.lsc-group.phys.uwm.edu/daswg/projects/ lal.html.

[12] E. Chassande-Mottin and A. Pai, Phys. Rev. D 73, 042003 (2006).

[13] E. J. Cands, P. R. Charlton, and H. Helgason, Classical Quantum Gravity 25, 184020 (2008).

[14] A. Buonanno, Y. Chen, and M. Vallisneri, Phys. Rev. D 67, 024016 (2003); 74, 029903 (2006). 